OPEN ACCESS

Edited by:

Daniel Zeller,

University of Würzburg, Germany

Reviewed by: Annapoorna Kuppuswamy, University College London, UK Matthias Grothe

University Greifswald, Germany

${ }^{*}$ Correspondence: Franca Tecchio,

LET'S Laboratory of

Electrophysiology for Translational neuroScience, ISTC-Consiglio Nazionale delle Ricerche (CNR) Unità MEG - Osp. Fatebenefratelli, Isola

Tiberina, 00186 Roma, Italy franca.tecchio@cnr.it

Specialty section: This article was submitted to Multiple Sclerosis and Neuroimmunology, a section of the journal Frontiers in Neurology

Received: 06 March 2015 Accepted: 11 June 2015 Published: 03 July 2015

Citation:

Tecchio F, Cancelli A, Cottone $C$, Ferrucci $R$, Vergari $M$, Zito $G$, Pasqualetti P, Filippi MM, Ghazaryan A, Lupoi D, Smits FM, Giordani A, Migliore S, Porcaro C, Salustri C, Rossini PM and Priori $A$

(2015) Brain plasticity effects of neuromodulation against multiple sclerosis fatigue.

Front. Neurol. 6:141. doi: 10.3389/fneur.2015.00141

\section{Brain plasticity effects of neuromodulation against multiple sclerosis fatigue}

\author{
Franca Tecchio ${ }^{1,2 *}$, Andrea Cancelli, ${ }^{1,3}$, Carlo Cottone ${ }^{1}$, Roberta Ferrucci ${ }^{4}$, \\ Maurizio Vergari ${ }^{4}$, Giancarlo Zito ${ }^{1,5}$, Patrizio Pasqualetti ${ }^{2,5}$, Maria Maddalena Filippi ${ }^{5}$, \\ Anna Ghazaryan ${ }^{1,5}$, Domenico Lupoi ${ }^{5}$, Fenne M. Smits ${ }^{6}$, Alessandro Giordani ${ }^{3,5}$, \\ Simone Migliore ${ }^{7}$, Camillo Porcaro ${ }^{1,8}$, Carlo Salustri ${ }^{1}$, Paolo M. Rossini ${ }^{2,3}$ and \\ Alberto Priori ${ }^{4}$
}

${ }^{1}$ Laboratory of Electrophysiology for Translational neuroScience (LET'S), Department of Neuroscience, ISTC, CNR, Fatebenefratelli Hospital - Isola Tiberina, Rome, Italy, ${ }^{2}$ Unit of Neuroimaging, IRCCS San Raffaele Pisana, Rome, Italy, ${ }^{3}$ Clinical Neurology, Catholic University, Policlinico A. Gemelli, Rome, Italy, ${ }^{4}$ Fondazione IRCCS Ca' Granda, Ospedale Maggiore Policlinico and Università degli Studi di Milano, Milan, Italy, ${ }^{5}$ AFaR Division, Fatebenefratelli Foundation for Health Research and Education, Rome, Italy, ${ }^{6}$ University of Amsterdam, Amsterdam, Netherlands, ${ }^{7}$ University of Campus Biomedico, Psychology Service, Rome, Italy, ${ }^{8}$ Institute of Neuroscience, Medical School, Newcastle University, Newcastle upon Tyne, UK

Rationale: We recently reported on the efficacy of a personalized transcranial direct current stimulation (tDCS) treatment in reducing multiple sclerosis (MS) fatigue. The result supports the notion that interventions targeted at modifying abnormal excitability within the sensorimotor network could represent valid non-pharmacological treatments.

Objective: The present work aimed at assessing whether the mentioned intervention also induces changes in the excitability of sensorimotor cortical areas.

Method: Two separate groups of fatigued MS patients were given a 5-day tDCS treatments targeting, respectively, the whole body somatosensory areas $\left(\mathrm{S}_{1}{ }_{\mathrm{wb}}\right)$ and the hand sensorimotor areas (SM1 hand). The study had a double blind, sham-controlled, randomized, cross-over (Real vs. Sham) design. Before and after each treatment, we measured fatigue levels (by the modified fatigue impact scale, mFIS), motor evoked potentials (MEPs) in response to transcranial magnetic stimulation and somatosensory evoked potentials (SEPs) in response to median nerve stimulation. We took MEPs and SEPs as measures of the excitability of the primary motor area (M1) and the primary somatosensory area (S1), respectively.

Results: The Real S1 $1_{w b}$ treatment produced a $27 \%$ reduction of the mFIS baseline level, while the $S M 1$ hand treatment showed no difference between Real and Sham stimulations. M1 excitability increased on average $6 \%$ of the baseline in the $S 1_{\text {wb }}$ group and $40 \%$ in the $\mathrm{SM}_{1}$ hand group. Observed SEP changes were not significant and we found no association between M1 excitability changes and $\mathrm{mFIS}$ decrease.

Conclusion: The tDCS treatment was more effective against MS fatigue when the electrode was focused on the bilateral whole body somatosensory area. Changes in S1 and M1 excitability did not correlate with symptoms amelioration. 
Significance: The neuromodulation treatment that proved effective against MS fatigue induced only minor variations of the motor cortex excitability, not enough to explain the beneficial effects of the intervention.

Keywords: fatigue in multiple sclerosis, electroencephalography, transcranial magnetic stimulation, transcranial direct current stimulation, magnetic resonance imaging, electrode personalization

\section{Introduction}

Fatigue is defined as "a feeling of insufficient physical and/or mental energies interfering with the usual and desired activities" (1). It is a common and highly disabling symptom in patients affected by multiple sclerosis (MS) even when other symptoms remain mild (2).

\section{Involvement of the Motor Control System in MS Fatigue}

To date, there is no clear evidence pointing at a single factor causing MS fatigue and fatigue complaints appear completely unrelated to both clinical variables, such as type of MS, level of disability, or disease duration, and demographic ones, such as age, gender, and education level (3). Although peripheral conditions, such as muscle weakness, may play a role, there are clear indications that much of MS fatigue has a central origin, most likely being the consequence of a failing central motor transmission to spinal alpha motor neurons (4).

\section{tDCS Treatment Targeting "Whole Body S1" vs. "Hand SM1"}

A few years ago, Cogiamanian obtained an increase of endurance against fatigue in healthy subjects by submitting them to a transcranial direct current stimulation (tDCS) (5). Recently, we applied Cogiamanian's treatment to fatigued MS patients (6) obtaining a significant amelioration of their symptoms. In the present study, we tested two variations of Cogiamanian's protocol on two distinct subgroups of fatigued MS patients. We submitted the first subgroup to Cogiamanian's same treatment only replacing the original mono-hemispheric with a bihemispheric stimulation (we will call this treatment $\mathrm{SM} 1_{\text {hand }}$ ).

It is known that fatigued MS patients show a much higher excitability of their primary motor area (M1) than non-fatigued patients and healthy subjects. This phenomenon has been attributed to a failure of intracortical inhibition (ICI) in frontal and M1 areas, both before and after fatiguing exercises (4). Furthermore, structural and functional data report a parietal involvement in MS fatigue symptoms (7-9), with indications of a reduced primary somatosensory area (S1) excitability $(10,11)$, and tDCS has been reported to enhance parieto-frontal projections (12). Also, in previous works of ours, we noticed signs of impaired communication between S1 and M1 (13).

Consequently, on the base of the above considerations, for the second subgroup, we modified Cogiamanian's treatment to selectively direct our neuromodulation on bihemispheric whole body S1, avoiding further direct enhancement of M1 excitability (14). Cogiamanian and coworkers assessed fatigue in hand movements and stimulated the hand section of SM1 representation (5). We considered that in MS patients, the lower limbs are also primarily involved and there are no reasons to limit neuromodulation to only the section of S1 devoted to hand representation. Thus, we treated the second subgroup with a tDCS on bilateral whole body $\mathrm{S} 1$ (we will call this treatment $S 1_{\mathrm{wb}}$ ).

\section{Aim}

Within this theoretical frame, our present aim was to test whether a tDCS treatment, which decreases MS fatigue, induces changes in brain excitability. In particular, we intended to quantify the effects induced within M1 via transcranial magnetic stimulation (TMS)evoked motor evoked potentials (MEPs) (15) and in S1 via median nerve $(\mathrm{MN})$ evoked somatosensory evoked potentials (SEPs).

\section{Materials and Methods}

The protocol was approved by the Ethics Committee of the "S. Giovanni Calibita" Fatebenefratelli Hospital in Rome and by Ethics Committee of Università degli Studi di Milano, Fondazione IRCCS Ospedale Maggiore Policlinico, Mangiagalli e Regina Elena.

\section{Study Design}

Both our studies ( $S 1_{\mathrm{wb}}$ and $\mathrm{SM} 1_{\text {hand }}$ treatments) followed a double blind, sham-controlled, randomized, cross-over design (Sham/Real, Real/Sham). Patient remained blind to whether they would receive a real or a sham treatment. Patients were asked to fill out the modified fatigue impact scale (mFIS) form to score their level of fatigue. We will refer to the week before the first tDCS treatment as T0 (baseline) and to at least $4 \mathrm{~h}$ after the last tDCS treatment as T1. We collected electroencephalographic (EEG) and TMS sessions and mFIS scores at T0 and T1.

\section{Sample Size Estimate}

We calculated the sample size using the repeatability of mFIS scores before neuromodulation treatments started. In 10 individuals with mild MS, we collected mFIS twice, 1 week apart. The average mFIS pre-post score difference was $0.1 \pm 1.9$, and the IntraClass Correlation indicated a very high agreement $(\mathrm{ICC}=0.96$; $p<0.001)$. According to our previous study (6), the variability of changes after stimulation was quite larger $(21.1 \%$ after real, $16.9 \%$ after sham). In order to assume the "worst" yet more realistic scenario, we did not lean on homoscedasticity and assumed both such variability values, distinguishing real and sham variances of pre-post-stimulation changes. In Tecchio et al. (6), we observed a 27\% improvement after real and 7\% improvement after sham treatment. To recognize as significant (alpha level $=0.05$ ), a $20 \%$ difference between Real and Sham treatments, a sample size of 10 cases will provide a power of $90 \%$. Notably, biomedical literature considers a $25 \%$ improvement (ere expected for Real stimulation) as a suitable threshold of clinical relevance (16) and here would 
correspond to a decrease of $12 \mathrm{mFIS}$ points for a severely fatigued patient with 48 at baseline.

\section{Participants}

We recruited 21 relapsing-remitting (RR) MS patients (17) experiencing fatigue [physical items mFIS score $>15$, Ref. (18)]. Inclusion criteria were as follows: mild physical disability [expanded disability status scale, EDDS (19) cut-off score of $\leq 3$ ], absence of depression (no pharmacological treatment), absence of clinical relapse, or radiological evidence of disease activity over the last 3 months. Exclusion criteria were as follows: use of symptomatic drugs, which may affect the level of fatigue, depression, and anxiety within the past 3 months (20), epilepsy or other central/peripheral nervous system comorbidities and any systemic conditions, which may cause fatigue (e.g., anemia and pregnancy). All patients underwent brain magnetic resonance imaging (MRI) for exclusion criteria assessment. In addition, a detailed clinical history was collected including active disease modifying therapy (DMT), disease duration, annual relapse rate, and depression level (Beck depression inventory, BDI). Fine hand motor control was evaluated by nine hole peg test (9HPT) scores collected separately for left and right sides.

\section{MRI Exam and Measure Estimate Image Acquisition}

In each patient undergoing $S 1_{\mathrm{wb}}$ treatment, brain imaging was performed by an Achieva 1.5-T scanner (Philips Medical Systems, Best, The Netherlands), with $33 \mathrm{mT} / \mathrm{m}$ gradients, online 2D/3D geometric distortion correction, and an 8-channels head Phased-Array coil with parallel imaging capabilities (SENSE). All sequences were acquired with contiguous slices and full brain coverage.

Exclusion criteria (no active lesions) were assessed based on T1-Spin Echo images before and 5 min after intravenous injection of a contrast agent. Lesions estimates were based on T2 Dual Echo images (see: column 2 of Attachment 2) and 3D-FLAIR (see: column 4 of Attachment 2).

T1-3D Fast Field Echo sequences with full brain coverage (MPRAGE, TR/TE/FA $=8.6 \mathrm{~ms} / 4 \mathrm{~ms} / 8^{\circ} ; 170$ contiguous sagittal slices $1.2 \mathrm{~mm}$ thick without gap, $\mathrm{mtx} 1922$ ) were used for the $3 \mathrm{D}$ reconstruction of the brain structure in order to personalize the tDCS electrode.

\section{Image Post-Processing Computations Lesion load}

A semi-automated region of interest (ROI) approach was used to trace hyperintense lesions in the white matter (WM) on T2weighted images, following strategies previously described [Ref. (11); Jim 5.0, Xinapse Systems Ltd., Leicester, UK, Attachment 3]. ROIs were identified by consensus of two investigators (Giancarlo Zito and D. Lupoi) blind to patients' clinical data. The total lesion volume (TLV) was computed. Lesion relative fraction ( $\mathrm{LrF}$ ) was computed as the ratio of the TLV over the WM volume in order to normalize for inter-subject head volume variability.

\section{Whole Body S1 Personalized Electrode Shaping and Positioning Personalized Electrode Shaping}

A few days before the experimental session, each subject underwent a structural brain MRI exam with a 1.5-T scanner (Achieva, Philips Medical Systems, Best, Netherlands; MPRAGE contiguous sagittal slices with full brain coverage). MRI data were elaborated with SofTaxic Neuronavigation System ver. 2.0 (www. softaxic.com, E.M.S., Bologna, Italy), which delivered the volumetric reconstruction of the individual brains and the cortical folders. The stereotaxic procedure for the personalization of each electrode included the following steps [Figure 1; (14)]: (1) the line of the central sulcus shown by the navigator is manually transferred onto a paper sheet firmly fixed onto the patient's scalp; (2) on paper, $2 \mathrm{~cm}$-long segments are drawn perpendicularly from a number of equidistant points of the central sulcus line in the anterior direction. The number of equidistant points is chosen to obtain a total electrode surface of $35 \mathrm{~cm}^{2}$, which the literature widely reports as the recommended size for a direct current intensity of $1.5 \mathrm{~mA}$. (3) The shape obtained on paper is transferred onto a commercial band of conductive silicone. The latter is $0.2 \mathrm{~mm}$ thick and has a $1 \mathrm{~mm}$ diameter channel running along its length. The electrode is manually cut along the contour, making sure that the channel remains roughly at the center of the band's length. (4) A standard electric wire, which will deliver the $1.5 \mathrm{~mA}$ direct current, is finally placed inside the channel.

Following the SofTaxic navigator, the electrode was positioned $1.5 \mathrm{~cm}$ posterior and $0.5 \mathrm{~cm}$ anterior to the central sulcus, centered on the nasion-inion line. Cathode electrode $(6 \mathrm{~cm} \times 14 \mathrm{~cm})$ was

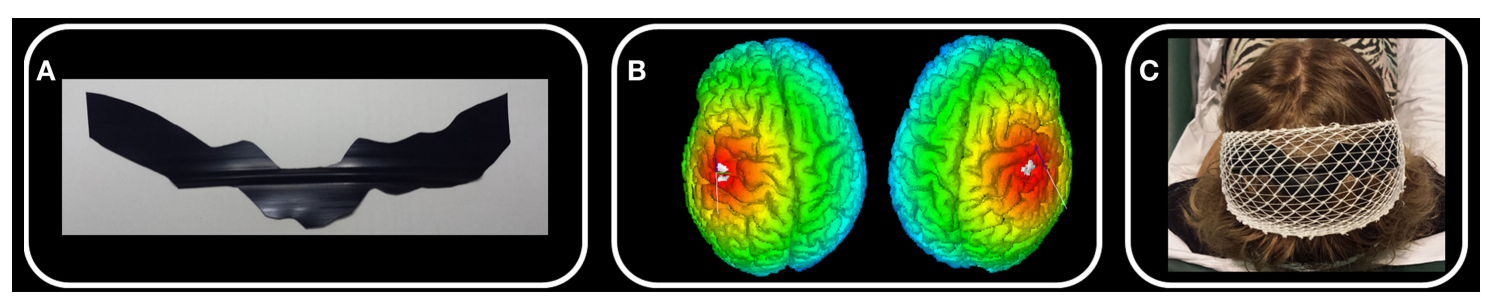

FIGURE 1 | Whole body S1 personalized electrode. In one exemplificative subject, we schematize the main steps of electrode personalization [Ref. (14), see Materials and Methods). (A) After drawing the left and right central sulci using SoftTaxic software from individual 3D $\mathrm{MRI}$, we fit this line by $2 \mathrm{~cm}$ wide parallelograms and we cut the electrode from a conductive silicon band. (B) We position the personalized stimulating electrode by proper neuronavigation procedure along the central sulcus with the center of the electrode crossing the nasion-inion line. (C) $S 1_{w b}$ personalized electrode and the cathode electrode positioned on $\mathrm{Oz}$. 
positioned on Oz. Contact with the subject's head was facilitated by a conductive gel and an elastic cotton net maintained the electrodes stable along the entire session (Figure 1).

\section{Transcranial Direct Current Stimulation (5-Day Treatment)}

Transcranial direct current stimulation was delivered by an electrical stimulator through a constant current unit and an isolation unit [SM1 (21); S1-Eldith Stimulator by NeuroConn, Ilmenau, Germany]. Anode electrode was positioned as described above. Cathode electrode was under the chin for the SM1 stimulation and on $\mathrm{Oz}$ for the $\mathrm{S} 1$ stimulation.

The 1.5-mA constant current was applied for 15 min once a day for five consecutive days, according to previous studies against pain $(22,23)$. In particular, a $1.5-\mathrm{mA}$ current strength produces a current density of about $0.04 \mathrm{~mA} / \mathrm{cm}^{2}$ for the anode electrode of $35 \mathrm{~cm}^{2}(5,24)$, which is well below safety thresholds. Cathode electrode size was of $84 \mathrm{~cm}^{2}$, resulting in a current density of $0.02 \mathrm{~mA} / \mathrm{cm}^{2}$ under this electrode, corresponding to a non-effect current density in this reference region $(25,26)$. Impedances were below $10 \mathrm{k} \Omega$ throughout the stimulations. Sham condition consisted of $4 \mathrm{~s}$ of active stimulation at the beginning and at the end of each day's 15-min stimulation. At debriefing, no subject reported to feel any difference across tCSs.

\section{Transcranial Magnetic Stimulation to Probe Cortical Excitability Changes in M1}

Single-pulse TMS was performed through a standard focal coil (diameter of each wing $70 \mathrm{~mm}$ ) connected with a Bistim 200 module (The Magstim Company Ltd., Whitland, UK). We recorded TMS MEPs from left and right opponens pollicis (OP) by surface electrodes in a belly tendon montage $(2.5 \mathrm{~cm}$ apart). Following international standards, we identified the "hot-spot" of the right OP muscle and the corresponding resting motor threshold (RMT) $(27,28)$. Thereafter, we maintained the coil position - digitized and monitored throughout the whole session by the SofTaxic neuronavigator - by means of a support arm (Figure 2A).
Transcranial magnetic stimulation intensity was settled at $120 \%$ RMT and 20 MEPs were then collected in complete relaxation while TMS was delivered with an inter-stimulus interval randomly ranging between 5 and $7 \mathrm{~s}$. The whole procedure was repeated in the other hemisphere to obtain left OP motor cortical representation.

\section{Electroencephalographic Study to Probe Cortical Excitability Changes in S1 \\ Electrophysiological Data Recording}

Electroencephalographic signals were recorded with a 64-channel actiChamp System (Brain Products GmbH, Gilching, Germany, Figure 2B). The montage included $\mathrm{Fz}$ derivation for reference and $\mathrm{FPz}$ for ground. EEG signals were sampled at $5 \mathrm{kHz}$ and a preconditioning $0.1-1500 \mathrm{~Hz}$ bandpass filtering was applied.

\section{Median Nerve Stimulation}

All subjects sat comfortably on an armchair during the experiment. In order to induce somatosensory evoked responses following a painless thumb twitch, their MN was stimulated at the wrist with a constant current electrical stimulator (Model DS3, Digitimer Ltd., Hertfordshire, UK), using standard parameters (cathode proximal, $250 \mathrm{~ms}$ inter-stimulus interval, $0.2 \mathrm{~ms}$ duration, above motor threshold intensity).

Left and right MNs were separately stimulated for $5.5 \mathrm{~min}$, totaling about 1300 artifact-free trials, which were stored for off-line analysis. The SEP epochs ranged from $10 \mathrm{~ms}$ pre to $100 \mathrm{~ms}$ post-stimulus. Epochs whose voltage amplitudes exceeded $\pm 100 \mu \mathrm{V}$ at the EOG electrode as well as those containing saturating artifacts were rejected.

All amplitude values referred to the $5-10 \mathrm{~ms}$ post-stimulus interval. The amplitude of the N20 component was measured as the first negative peak between 18 and $23 \mathrm{~ms}$. The N20-P25 complex was determined as the difference between the N20 peak and the subsequent positivity peak (P25), occurring at a latency of around 23-29 ms.

For purposes of the present study, we used the typical bipolar derivation used to assess SEPs [C3-C4, Ref. (29)].

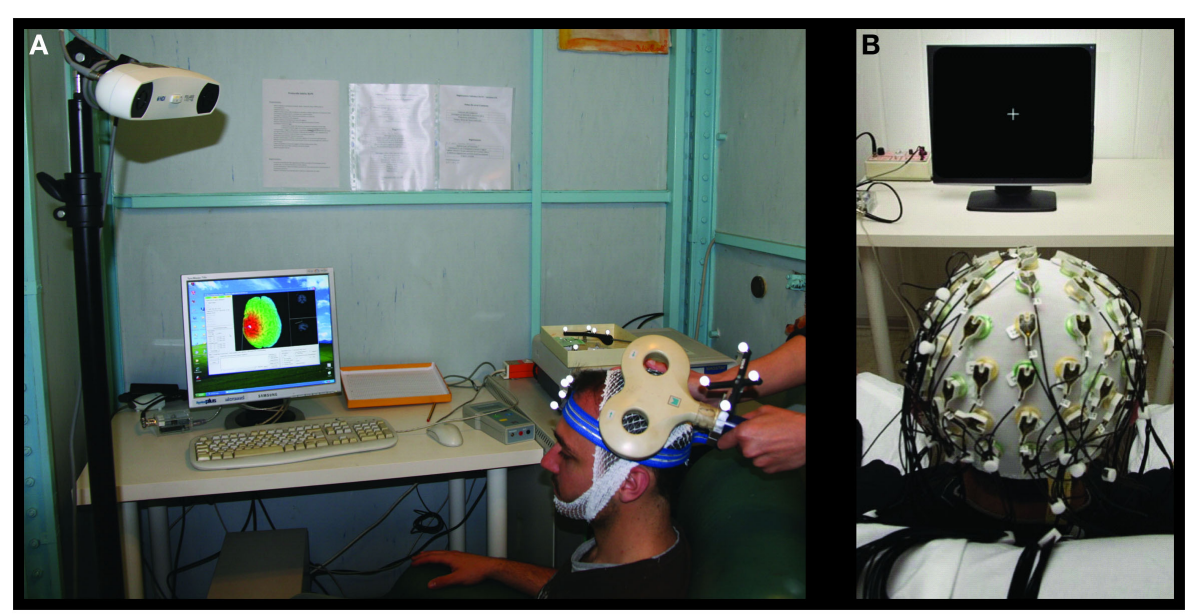

FIGURE 2 | Transcranial magnetic stimulation and EEG settings for brain plasticity assessment. Experimental settings for the MEP (A) and SEP (B) recordings. 


\section{Statistical Analysis}

After checking the distribution of MEP and SEP amplitudes (as tested by Shapiro-Wilk test), we applied, when necessary, suitable transformations in order to achieve a better approximation to gaussianity and a good control of outliers.

To test the effects of the 5-day tDCS on MFIS, MEP, and SEP variables, analyses of variance (ANOVA) for repeated measures were performed with Stimulation (Real, Sham) and Treatment (pre-, post-tDCS treatment) as within-subjects factors. Withinsubjects factor Hemisphere (Left, Right) was included for MEP and SEP, which had been collected bilaterally since we performed a bilateral stimulation. A similar approach was used for the effects on fine hand motor control measure, with the 9HPT submitted to the ANOVA with $t D C S$ Intervention (Pre, Post), Stimulation (Real, Sham), and Hand (Right, Left) within-subjects factors. We performed separate ANOVA designs in the two patients' subgroups stimulated on bilateral $S 1_{\mathrm{wb}}$ or $S M 1_{\text {hand }}$. Significance threshold was set to 0.050 and we reported trends for $p<0.100$.

\section{Results}

The 21 patient cohorts presented a mild clinical picture in accordance to the inclusion criteria (Table 1). The two electrodedependent subgroups displayed homogenous clinical features (Table 1).

\section{Fatigue Levels Whole Body $\mathbf{S 1}$ Stimulation $\left(\mathbf{S} \mathbf{w b}_{\mathrm{wb}}\right)$}

Analyses of variance indicated that $\mathrm{mFIS}$ changes were related to the type of stimulation (Real or Sham) when the bilateral personalized $\mathrm{S}_{\mathrm{wb}}$ electrode was used [Stimulation $\times$ Treatment interaction $F(1,8)=9.692, p=0.014$, (6), Table 2]. Fatigue resulted reduced after real stimulation (post hoc comparison $p=0.002$, $31.0 \pm 12.0$ post- vs. $42.1 \pm 7.9$ pre-stimulation), whereas there were no changes after the sham stimulation [post hoc comparison $p=0.901,34.8 \pm 10.4$ post- vs. $37.2 \pm 7.0$ pre-stimulation, (6), Table 2]. After real stimulation, the mean fatigue reduction was $28 \%$ of the baseline (range between 2 and $76 \%$ ), and $8 \%$ after sham (range between -11 and $38 \%$, paired-samples $t$-test real vs. sham, $p=0.016)$.

TABLE 1 | Demographic and clinical profile of people with MS.

\begin{tabular}{lllllllll}
\hline & Sex & Age & Dis Dur & EDSS & BDI & mFIS & LrF & 9HPT \\
\hline $\mathrm{S}_{\text {wb }}$ & $9 \mathrm{~F} / 4 \mathrm{M}$ & 45.8 & 7.6 & 1.5 & 12.7 & $41.6^{\mathrm{a}}$ & 0.38 & 20.8 \\
& & $(7.6)$ & $(8.2)$ & {$[0-3.5]$} & $(3.5)$ & $(7.5)^{\mathrm{a}}$ & $(0.48)$ & $(4.9)$ \\
$\mathrm{SM}_{\text {hand }}{ }^{\mathrm{b}}$ & $6 \mathrm{~F} / 2 \mathrm{M}$ & 38.1 & 13.5 & 2 & 11.0 & 57.1 & & \\
& & $(9.8)$ & $(4.2)$ & {$[1-2.5]$} & $(5.1)$ & $(19.9)$ & & \\
& $p$ & 0.080 & 0.068 & 0.254 & 0.438 & 0.062 & &
\end{tabular}

$M$, male; F, female; Mean or Median in italics and $S D$, standard deviations 0 or ranges [min, max] across the group of: Dis Dur, disease duration; Scores of: EDSS, expanded disability status scale; $B D I$, Beck depression inventory; $L r F$, lesion relative factor; MFIS, modified fatigue impact scale, and 9HPT, time (s) to execute right hand 9-Hole Peg Test at baseline.

${ }^{a}$ mFIS 1-week apart repetition was 41.5, SD 6.1 (see Study Design). MRI-derived measures ( $\mathrm{LrF}$ ) and 9HPT were not collected in the SM1 hand group.

${ }^{b}$ Two out of the 10 patients of the SM1 hand group dropped out. In the last row, the significance og the comparison between the two groups.
TABLE 2 | Transcranial direct current stimulation treatment effects on fatigue.

\begin{tabular}{|c|c|c|c|c|c|}
\hline & \multicolumn{2}{|c|}{ Real } & \multicolumn{2}{|c|}{ Sham } & \multirow[b]{2}{*}{$p$} \\
\hline & TO & T1 & TO & T1 & \\
\hline$S 1_{w b}$ & $\begin{array}{l}\mathbf{4 2 . 1} \\
(7.9)\end{array}$ & $\begin{array}{l}\mathbf{3 1 . 0} \\
(12.0)\end{array}$ & $\begin{array}{l}37.2 \\
(7.0)\end{array}$ & $\begin{array}{l}34.7 \\
(10.4)\end{array}$ & 0.014 \\
\hline$S M 1_{\text {hand }}$ & $\begin{array}{l}57.8 \\
(19.9)\end{array}$ & $\begin{array}{l}42.1 \\
(17.2)\end{array}$ & $\begin{array}{l}55.5 \\
(26.6)\end{array}$ & $\begin{array}{l}52.1 \\
(22.0)\end{array}$ & 0.239 \\
\hline
\end{tabular}

Mean and SD of fatigue scale (mFIS) across patients before and after 5-day tDCS treatment, stimulating bilateral either whole body $S 1$ ( $\left.S 1_{w b}\right)$ or hand section of SM1 $\left(S M 1_{\text {hand }}\right) . p$ is the significance of the Stimulation $\times$ Treatment interaction effect. In bold, values with significant difference between pre- and post-treatment, as estimated by post-hoc comparison whenever the Stimulation $\times$ Treatment interaction effect was significant.

\section{tDCS Treatment Effect on Fine Hand Motor Control (9HPT)}

In the $S 1_{\mathrm{wb}}$ group, 9HPT of the right hand correlated with both EDSS and physical items of MFIS (Pearson's $r=0.736, p=0.015$ and $r=0.744, p=0.014$, respectively). It should be noted that the correlation between MFIS_phys and 9HPT remains substantially stable after correction for EDSS (partial correlation $r=0.602$, $p=0.086$ ). The lesion load was not associated with any clinical or fatigue-related measure (LrF with EDSS, BDI, total or physical MFIS $p>0.200$ consistently).

The full model ANOVA evidenced, in addition to the right hand performing better than the left [Hand factor $F(1,8)=5.749$, $p=0.043$, overall average $20.3 \pm 4.6$ and $22.6 \pm 4.3 \mathrm{~s}$, respectively], that the two hands' performances were differently affected by the intervention [Hand (Right, Left) $\times t D C S$ intervention (Pre, Post $) \times$ Stimulation (Real, Sham) effect $F(1,8)=5.697, p=0.044]$. Repeating the reduced models for each hand separately, we observed that the left hand did not change after the 5-day stimulation, while the right hand 9HPT changed in terms of dependence on whether the stimulation was real or sham [Stimulation $\times t D C S$ intervention effect $F(1,8)=5.680, p=0.044]$. The post hoc comparison showed that, after the real stimulation, the time required to execute the 9HPT decreased (two-tails paired $t$-test $p=0.038$, with average $21.1 \pm 4.9$ pre and $19.8 \pm 3.8$ s post values], while it was unchanged by sham stimulation $(t$-test $p=0.401)$. No association emerged among post-tDCS values of MFIS regarding either total or physical and 9HPT scores.

\section{Hand SM1 Stimulation (SM1 ${ }_{\text {hand }}$ )}

No interaction Stimulation $\times$ Treatment effect was observed when $S M 1_{\text {hand }}$ electrode was used ( $p>0.200$, Table 2), indicating that effects of real and sham stimulations on fatigue levels were not clearly different.

\section{M1 Excitability}

No differences were observed in RMTs, stimulation intensities or MEP latencies when compared between hemispheres, between stimulation types (Real or Sham) or treatments (pre-poststimulation) ( $p>0.200$ consistently). In the $S 1_{w b}$ group, the mean of RMT across all conditions was $58.4 \pm 2.6 \%$ of the maximal stimulator output, TMS intensity was $70.2 \pm 3.1 \%$, MEP latency 
was $27.3 \pm 3.2 \mathrm{~ms}$. In the $\mathrm{SM}_{1}$ hand group, the RMT decreased after stimulation (paired-samples $t$-test $p=0.004$; pre $59.2 \pm 18.8$ and post $55.0 \pm 17.9 \%$ of the maximal stimulator output), while the latency did not change $24.4 \pm 2.6 \mathrm{~ms}$. MEP latency was associated to the MS severity (EDSS-MEP latency Pearson's coefficient $r=0.880, p=0.021$ ).

Motor evoked potential amplitude distribution definitely differed from a Gaussian and we obtained a good fit by natural logarithmic transformation (Shapiro-Wilk $p>0.200$ consistently).

No association was evident between the order of MEP collection and its amplitude (Pearson's correlation $p=0.607$ ). Mean MEP amplitude, estimated as exponential backtransformation of mean of logarithm-transformed MEP amplitudes, were $171.5 \pm 1.8$ for the right OP and $145.3 \pm 1.9$ for the left OP. No difference of baseline MEP amplitudes was observed between Real and Sham stimulations ( $t$-test $p=0.380$ averaging right and left values).

In the $S 1_{w b}$ group, the ANOVA on the MEP amplitude showed a trend interaction effect Stimulation $\times$ Treatment $(p=0.073)$, which corresponded to an increase of MEP amplitude after the real stimulation (Treatment effect, $p=0.037$ ), absent after Sham $(p=0.275)$. The average increase with respect to the baseline level was $6.0 \%$ ranging between 0.2 and $22.6 \%$ of baseline level (MEPpost-MEPpre/MEPpre of logarithm-transformed MEP amplitudes averages).

In the $\mathrm{SM} 1_{\text {hand }}$ group, MEP amplitude increased after the real stimulation (Treatment effect, $p=0.021$ ). The average increase with respect to the baseline level was $40.4 \%$ ranging between 16.7 and $76.0 \%$ of baseline level.

No association emerged among post-tDCS MEP values and 9HPT scores.

\section{S1 Excitability}

No effects were observed in the N20 SEP component between the two hemispheres or the two stimulations (pre-post-stimulation, Real or Sham, $p>0.200$ consistently). The N20-P25 complex showed a tendency to increasing after the stimulation, but no Stimulation $\times$ Treatment effect was found $(p>0.200)$.

\section{Relationships Between S1 and M1 Excitability and MFIS Variations}

Fatigue level changes did not correlate with variations in M1 excitability in either of the $S 1_{\mathrm{wb}}$ or $\mathrm{SM} 1_{\text {hand }}$ subgroups $(p>0.200$ in both cases).

\section{Discussion}

Our 5-day tDCS stimulation targeting the bihemispheric whole body somatosensory region significantly decreased MS fatigue. In addition, hand muscle MEPs showed that that stimulation modified M1 excitability, whereas MN SEPs showed no evidence of changes in S1 excitability.

\section{Mechanisms Behind Regional Dependence of tDCS Treatment Efficacy (i.e., S1-Whole Body vs. SM1-Hand)}

Overall, the 5-day tDCS treatment targeting the bilateral $S 1_{\mathrm{wb}}$ representation showed the Stimulation $\times$ Treatment effect, which
TABLE 3 | Intra-cohort fatigue levels correlation.

\begin{tabular}{|c|c|c|c|c|}
\hline & \multicolumn{2}{|c|}{$\mathbf{S} 1_{\mathrm{wb}}$} & \multicolumn{2}{|c|}{$S M 1_{\text {hand }}$} \\
\hline & $\rho$ & $p$ & $\rho$ & $p$ \\
\hline Real vs. Sham T0 & 0.718 & 0.045 & -0.299 & 0.471 \\
\hline Real T0 vs. T1 & 0.840 & 0.002 & 0.403 & 0.323 \\
\hline Sham T0 vs. T1 & 0.957 & 0.000 & -0.054 & 0.900 \\
\hline
\end{tabular}

Pearson's correlation coefficients ( $\rho$ ) and significance $(p)$ between real and sham baseline mFIS scores (TO) and between mFIS scores at baseline (TO) and after-treatment (T1) both after real (second row) and sham (third row) treatments, for both the $S M 1_{\text {hand }}$ and $S 1_{\text {wb }}$ subgroups. In bold, significant correlations.

was lacking in the $\mathrm{SM} 1_{\text {hand }}$ intervention. Noteworthy, the average decrease in mFIS score was 15.6 after real $S M 1_{\text {hand }}$ stimulation - larger than the 11.1 point decrease observed after real $S 1_{w b}$ stimulation, with similar baseline levels - and the specific paired $t$ test comparison was significant $(p=0.030)$. We also analyzed why an average net (real minus sham) SM1 effect of 15.6-3.4=12.2 was not significant while an average net $\mathrm{S} 1$ effect of $11.1-2.4=8.7$ was significant, with comparable SD (a little bit larger in S1, indeed) (although such a comparison is irrelevant in the absence of a significant interaction). The reason lies in the different correlation patterns: within the $S M 1_{\text {hand }}$ subgroup, correlations are absent between Sham and Real baseline levels, as well as between fatigue scale values from other time points, contrasting clear (and expected) correlations in the $S 1_{\mathrm{wb}}$ subgroup (Table 3 ).

The $S 1_{\mathrm{wb}}$ treatment was more effective than over $\mathrm{SM} 1_{\text {hand }}$ (21) and than over the left prefrontal cortex (30). This comparative result strengthens the working hypothesis, which guided the development of the S1-whole body personalized electrode. In fact, data available in the literature document a failure of the inhibitory mechanisms in the frontal and primary motor (M1) areas involved in motor planning (4), a reduced M1 ICI before and after fatiguing exercises (4), and an increase in M1 excitability (4) in fatigued vs. non-fatigued MS patients and to healthy subjects. Concurrently, together with excessive excitability of $\mathrm{M} 1$, we observed signs of a reduced S1 excitability (10). Moreover, we observed an altered parieto-frontal projection, mainly involving S1 and M1, in fatigued vs. non-fatigued MS patients $(13,31)$. Thus, we decided to neuromodulate to enhance selectively the excitability of S1, avoiding a direct enhancement of M1 excitability (as occurs with SM1 electrode), to further support the parieto-frontal projection already observed by tDCS (12).

\section{Suitability of Differentiated Effects Targeting S1 vs. SM1}

Transcranial direct current stimulation-generated modulations of cortical excitability can be focused by means of proper sizing and positioning of the stimulation electrode. Since tDCS efficacy is determined by the current density (i.e., current strength/electrode area), we can obtain increased focality by reducing the electrode size while keeping a constant current strength. In the motor system, Nitsche and colleagues (32) compared tDCS effects on central representations of two muscles, first dorsal interosseus (FDI) and abductor digiti minimi (ADM), by measuring MEPs. Stimulation with small electrodes $\left(3.5 \mathrm{~cm}^{2}\right)$ generated focal effects, with different MEP amplitude increases for the two muscles (32). The protocol we are proposing actually requires less focality than 
Nitsche's, where a discrimination of M1 neuronal pools controlling the two hand muscles was sought. In fact, we intend to stimulate motor vs. sensory regions. However, while positioning of tDCS electrodes in M1 stimulation can be guided by TMS, which induces responses from specific muscles, a neuronavigation system is required when stimulating S1 vs. M1 to precisely identify the central sulcus. Modern frameless stereotaxic systems allow navigation on the subject's structural MRI-derived brain representation, providing high-spatial precision with accuracy in the range of millimeters (33). In our experimental setup, we used precise topographical determination of the central sulcus in placing the $S 1_{\mathrm{wb}}$ electrode $(6,14,25,34)$.

\section{tDCS Targeting Bilateral vs. Mono-Hemispheric Regions}

Multiple sclerosis fatigue is not associated to mono-hemispheric prevalence, as shown by electrophysiological (10) and neuroimaging data (9). Thus, via the tDCS intervention, we targeted bilateral (35) either whole body S1 or hand SM1. In the present results, we observed bilateral M1 enhancement, documenting that bilateral stimulations of a homologous area do not cancel out. This hypothesis of ineffective bilateral M1 stimulation is derived from the well-known motor system organization, with M1 of one hemisphere inhibiting M1 of the other hemisphere. Through bilateral stimulation of homologous M1 areas, the concurrent increase of inhibition induced by the increase in excitability of one hemisphere might thus cancel out the increase in excitability in the other hemisphere. However, we can reject such a hypothesis, and we can also speculate that a relevant component of the presently observed neuromodulation operates directly on local pyramidal neurons, and not via inhibitory or excitatory networks beneath the electrode $(36,37)$.

\section{Brain Plasticity Induced by S1 Stimulation}

We did not find evidence of S1 excitability changes induced by $S 1_{w b}$ tDCS treatment, as measured by the typical SEP assessment. This can be due to two causes. The first is that the SEP gives an indirect assessment of cortical pyramidal neurons with respect to

\section{References}

1. MSCfCP. Fatigue and Multiple Sclerosis: Evidence-Based Management Strategies for Fatigue in Multiple Sclerosis. Clinical Practice Guidelines. Washington, DC: Paralyzed Veterans of America (1998).

2. Krupp LB, Alvarez LA, LaRocca NG, Scheinberg LC. Fatigue in multiple sclerosis. Arch Neurol (1988) 45:435-7. doi:10.1001/archneur.1988.0052028008 5020

3. Krupp LB. Fatigue in multiple sclerosis: definition, pathophysiology and treatment. CNS Drugs (2003) 17:225-34. doi:10.2165/00023210-200317040-00002

4. Yusuf A, Koski L. A qualitative review of the neurophysiological underpinnings of fatigue in multiple sclerosis. J Neurol Sci (2013) 330:4-9. doi:10.1016/j.jns. 2013.04.012

5. Cogiamanian F, Marceglia S, Ardolino G, Barbieri S, Priori A. Improved isometric force endurance after transcranial direct current stimulation over the human motor cortical areas. Eur J Neurosci (2007) 26:242-9. doi:10.1111/j.1460-9568. 2007.05633.x

6. Tecchio F, Cancelli A, Cottone C, Zito G, Pasqualetti P, Ghazaryan A, et al. Multiple sclerosis fatigue relief by bilateral somatosensory cortex neuromodulation. J Neurol (2014) 261:1552-8. doi:10.1007/s00415-014-7377-9
TMS-derived MEP. In fact, TMS stimulates pyramidal neurons and the MEP muscle response gives a measure of cortical excitability with as a single-station-pathway (only the spinal cord relay in between). Instead, the pathway between $\mathrm{MN}$ stimulation and S1 (here assessed by single-derivation SEP) includes spinal cord, brain stem, and thalamic relays. The second reason can be poor sensitivity of EEG-derived SEP analysis. In addition, we found more effects in M1 than in S1, which can be due to non-selective S1 stimulation. Via simulations (in progress), we are in fact observing that the induced current density is slightly prevalent in S1 but it is of a comparable intensity also in M1.

\section{Study Limitations}

We did not study the two datasets $\left(\mathrm{S}_{\mathrm{wb}}\right.$ and $\mathrm{SM} 1_{\text {hand }} 5$-day tDCS treatments) in a single statistical model, since a different anode electrode size (anode electrode area of $70 \mathrm{~cm}^{2}$ for SM1 and $35^{2}$ for S1) and a different reference position (on $\mathrm{Oz}$ or on the left shoulder) were used.

Here, we investigated somatosensory evoked responses, since we performed a stimulation planned to focus on S1. We started from a standard single derivation in each hemisphere to assess SEP changes. Nevertheless, we collected 64-channel EEG data to further investigate cortical effects. In particular, source analysis will allow future investigations of our main hypothesis of a modification induced by the tDCS treatment on sensory-motor functional connectivity.

\section{Acknowledgments}

The authors are sincerely grateful to all patients for the time and cooperation offered during the study. This work was supported by: (1) FISM - Fondazione Italiana Sclerosi Multipla - Cod.2014/R [FaReMuS CuNeH], (2) Ministry of Health Cod. GR-2008-1138642 [ProSIA], (3) MIUR Prot. 2010SH7H3F "Functional connectivity and neuroplasticity in physiological and pathological aging [ConnAge]" and (4) PNR-CNR Aging Program 2012-2016.

7. Cruz Gomez AJ, Ventura Campos N, Belenguer A, Avila C, Forn C. Regional brain atrophy and functional connectivity changes related to fatigue in multiple sclerosis. PLoS One (2013) 8:e77914. doi:10.1371/journal.pone.0077914

8. Engstrom M, Flensner G, Landtblom AM, Ek AC, Karlsson T. Thalamostriato-cortical determinants to fatigue in multiple sclerosis. Brain Behav (2013) 3:715-28. doi:10.1002/brb3.181

9. Pellicano C, Gallo A, Li X, Ikonomidou VN, Evangelou IE, Ohayon JM, et al. Relationship of cortical atrophy to fatigue in patients with multiple sclerosis. Arch Neurol (2010) 67:447-53. doi:10.1001/archneurol.2010.48

10. Dell'Acqua ML, Landi D, Zito G, Zappasodi F, Lupoi D, Rossini PM, et al. Thalamocortical sensorimotor circuit in multiple sclerosis: an integrated structural and electrophysiological assessment. Hum Brain Mapp (2010) 31:1588-600. doi:10.1002/hbm.20961

11. Tecchio F, Zito G, Zappasodi F, Dell'Acqua ML, Landi D, Nardo D, et al. Intracortical connectivity in multiple sclerosis: a neurophysiological approach. Brain (2008) 131:1783-92. doi:10.1093/brain/awn087

12. Polania R, Nitsche MA, Paulus W. Modulating functional connectivity patterns and topological functional organization of the human brain with transcranial direct current stimulation. Hum Brain Mapp (2011) 32:1236-49. doi:10.1002/ hbm. 21104 
13. Tomasevic L, Zito G, Pasqualetti P, Filippi M, Landi D, Ghazaryan A, et al. Cortico-muscular coherence as an index of fatigue in multiple sclerosis. Mult Scler (2013) 19:334-43. doi:10.1177/1352458512452921

14. Tecchio F, Cancelli A, Cottone C, Tomasevic L, Devigus B, Zito G, et al. Regional personalized electrodes to select transcranial current stimulation target. Front Hum Neurosci (2013) 7:131. doi:10.3389/fnhum.2013.00131

15. Rossini PM, Zarola F, Floris R, Bernardi G, Perretti A, Pelosi L, et al. Sensory (VEP, BAEP, SEP) and motor-evoked potentials, liquoral and magnetic resonance findings in multiple sclerosis. Eur Neurol (1989) 29:41-7. doi:10.1159/ 000116376

16. Freiman JA, Chalmers TC, Smith H Jr, Kuebler RR. The importance of beta, the type II error and sample size in the design and interpretation of the randomized control trial. Survey of 71 "negative" trials. N Engl J Med (1978) 299:690-4. doi:10.1056/NEJM197809282991304

17. Lublin FD, Reingold SC, Cohen JA, Cutter GR, Sorensen PS, Thompson AJ, et al. Defining the clinical course of multiple sclerosis: the 2013 revisions. Neurology (2014) 83:278-86. doi:10.1212/WNL.0000000000000560

18. Kos D, Nagels G, D’Hooghe MB, Duportail M, Kerckhofs E. A rapid screening tool for fatigue impact in multiple sclerosis. BMC Neurol (2006) 6:27. doi:10. 1186/1471-2377-6-27

19. Kurtzke JF. Rating neurologic impairment in multiple sclerosis: an expanded disability status scale (EDSS). Neurology (1983) 33:1444-52. doi:10.1212/WNL. 33.11.1444

20. Rietberg MB, van Wegen EE, Uitdehaag BM, Kwakkel G. The association between perceived fatigue and actual level of physical activity in multiple sclerosis. Mult Scler (2011) 17:1231-7. doi:10.1177/1352458511407102

21. Ferrucci R, Vergari M, Cogiamanian F, Bocci T, Ciocca M, Tomasini E, et al. Transcranial direct current stimulation (tDCS) for fatigue in multiple sclerosis. NeuroRehabilitation (2014) 34(1):121-7. doi:10.3233/NRE-131019

22. Fregni F, Boggio PS, Lima MC, Ferreira MJ, Wagner T, Rigonatti SP, et al. A sham-controlled, phase II trial of transcranial direct current stimulation for the treatment of central pain in traumatic spinal cord injury. Pain (2006) 122:197-209. doi:10.1016/j.pain.2006.02.023

23. Mori F, Codeca C, Kusayanagi H, Monteleone F, Buttari F, Fiore S, et al. Effects of anodal transcranial direct current stimulation on chronic neuropathic pain in patients with multiple sclerosis. J Pain (2010) 11:436-42. doi:10.1016/j.jpain. 2009.08.011

24. Tecchio F, Zappasodi F, Assenza G, Tombini M, Vollaro S, Barbati G, et al. Anodal transcranial direct current stimulation enhances procedural consolidation. J Neurophysiol (2010) 104:1134-40. doi:10.1152/jn.00661.2009

25. Cancelli A, Cottone C, Zito G, Di Giorgio M, Pasqualetti P, Tecchio F. Cortical inhibition and excitation by bilateral transcranial alternating current stimulation. Restor Neurol Neurosci (2015) 33(2):105-14. doi:10.3233/RNN-140411

26. Moliadze V, Atalay D, Antal A, Paulus W. Close to threshold transcranial electrical stimulation preferentially activates inhibitory networks before switching to excitation with higher intensities. Brain Stimulat (2012) 5:505-11. doi:10. 1016/j.brs.2011.11.004

27. Rossini PM, Barker AT, Berardelli A, Caramia MD, Caruso G, Cracco RQ, et al. Non-invasive electrical and magnetic stimulation of the brain, spinal cord and roots: basic principles and procedures for routine clinical application. Report of an IFCN committee. Electroencephalogr Clin Neurophysiol (1994) 91:79-92. doi:10.1016/0013-4694(94)90029-9

28. Rossini PM, Burke D, Chen R, Cohen LG, Daskalakis Z, Di Iorio R, et al. Non-invasive electrical and magnetic stimulation of the brain, spinal cord, roots and peripheral nerves: basic principles and procedures for routine clinical and research application. An updated report from an I.F.C.N. committee. Neurophysiol Clin (2015) 126:1071-107. doi:10.1016/j.clinph.2015.02.001

29. Rossini PM, Basciani M, Di Stefano E, Febbo A, Mercuri N. Short-latency scalp somatosensory evoked potentials and central spine to scalp propagation characteristics during peroneal and median nerve stimulation in multiple sclerosis. Electroencephalogr Clin Neurophysiol (1985) 60:197-206. doi:10.1016/ 0013-4694(85)90031-8

30. Saiote C, Goldschmidt T, Timäus C, Steenwijk MD, Opitz A, Antal A, et al. Impact of transcranial direct current stimulation on fatigue in multiple sclerosis. Restor Neurol Neurosci (2014) 32(3):423-36. doi:10.3233/RNN- 130372

31. Cogliati Dezza I, Zito G, Tomasevic L, Filippi MM, Ghazaryan A, Porcaro $\mathrm{C}$, et al. Functional and structural balances of homologous sensorimotor regions in multiple sclerosis fatigue. J Neurol (2015) 262:614-22. doi:10.1007/ s00415-014-7590-6

32. Nitsche MA, Doemkes S, Karakose T, Antal A, Liebetanz D, Lang N, et al. Shaping the effects of transcranial direct current stimulation of the human motor cortex. J Neurophysiol (2007) 97:3109-17. doi:10.1152/jn.01312.2006

33. Sparing R, Buelte D, Meister IG, Paus T, Fink GR. Transcranial magnetic stimulation and the challenge of coil placement: a comparison of conventional and stereotaxic neuronavigational strategies. Hum Brain Mapp (2008) 29:82-96. doi:10.1002/hbm.20360

34. Cancelli A, Cottone C, Di Giorgio M, Carducci F, Tecchio F. Personalizing the electrode to neuromodulate an extended cortical region. Brain Stimul (2015) 8(3):555-60. doi:10.1016/j.brs.2015.01.398

35. Marshall L, Molle M, Siebner HR, Born J. Bifrontal transcranial direct current stimulation slows reaction time in a working memory task. BMC Neurosci (2005) 6:23. doi:10.1186/1471-2202-6-23

36. Artola A, Brocher S, Singer W. Different voltage-dependent thresholds for inducing long-term depression and long-term potentiation in slices of rat visual cortex. Nature (1990) 347:69-72. doi:10.1038/347069a0

37. Le Roux N, Amar M, Baux G, Fossier P. Homeostatic control of the excitationinhibition balance in cortical layer 5 pyramidal neurons. Eur J Neurosci (2006) 24:3507-18. doi:10.1111/j.1460-9568.2006.05203.x

Conflict of Interest Statement: The authors declare that the research was conducted in the absence of any commercial or financial relationships that could be construed as a potential conflict of interest.

Copyright (C) 2015 Tecchio, Cancelli, Cottone, Ferrucci, Vergari, Zito, Pasqualetti, Filippi, Ghazaryan, Lupoi, Smits, Giordani, Migliore, Porcaro, Salustri, Rossini and Priori. This is an open-access article distributed under the terms of the Creative Commons Attribution License (CC BY). The use, distribution or reproduction in other forums is permitted, provided the original author(s) or licensor are credited and that the original publication in this journal is cited, in accordance with accepted academic practice. No use, distribution or reproduction is permitted which does not comply with these terms. 